\title{
Male Bodily Responses During an Interaction with a Virtual Woman
}

\author{
Xueni Pan $^{1}$, Marco Gillies ${ }^{1}$, and Mel Slater ${ }^{1,2}$ \\ 1 University College London, London, UK \\ s.pan@cs.ucl.ac.uk, \\ 2 ICREA-Universitat Politécnica de Catalunya, Spain
}

\begin{abstract}
This work presents the analysis of the body movement of male participants, while talking with a life-size virtual woman in a virtual social encounter within a CAVE-like system. We consider independent and explanatory variables including whether the participant is the centre of attention in the scenario, whether the participant is shy or confident, and his relationship status. We also examine whether this interaction between the participant and the virtual character changes as the conversation progresses. The results show that the participants tend to have different hand movements, head movements, and posture depending on these conditions. This research therefore provides strong evidence for using body movement as a systematic method to assess the responses of people within a virtual environment, especially when the participant interacts with a virtual character. These results also point the way towards the application of this technology to the treatment of social phobic males.
\end{abstract}

\section{Introduction}

When a virtual character smiles at you, will you smile back, and why? In this research we consider whether people respond to virtual characters as if they were real, in the context of an interaction within an immersive virtual environment (VE). Our motivation is to assess the extent to which such environments can be used in place of physically real environments for studying issues such as social phobia $[7,10]$ and paranoia [3], and also treatment programmes for such social conditions. In previous work we have relied on questionnaire responses and physiological responses [7]. Here we consider the gestural and postural responses of male participants to an approach by an attractive and friendly virtual woman in a CAVE-like system.

In this paper, we discuss a new methodology where we examine participants' behavioural responses as a direct measure of the extent to which they respond as if the virtual encounter were real. Non-verbal behaviour (facial expression and body movements) reflects human beings' automatic responses. However, unlike verbal responses, body movement is an easily observable, gross overall indicator of a person's state. It therefore could offer us an additional method for assessing the realism of people's responses within a virtual environment. Moreover, while 
interacting with a virtual character, the participants' body movements reflect not only the states of the participants themselves but also how much are they engaged with the ongoing interaction with the virtual character.

Section 2 introduces the importance of using body movement as a measurement to VE. Section 3 describes the methodology. Results are presented in Section 4 and discussion in Section 5. The conclusions are given in Section 6.

\section{Related Work}

A common framework for measuring VE is "presence" which may be defined as the extent to which participants act and respond as if what they experience in the virtual reality were real [8]. This tendency of acting realistically towards virtually generated sense data distinguishes VE from all other media, such as films and books leading to a range beneficial applications from virtual psychotherapy to training. Therefore, instead of using only subjective responses as obtained from questionnaires and interviews in evaluating how people behave and respond within a VE, our work emphasises the importance of participants' measurable responses.

Most previous research on presence has used questionnaire and post-experiment interviews as the measurement instrument $[13,16]$. However, the participant can only complete the questionnaire and interviews after the experiment, therefore only reflecting what is in their memory. Moreover, as subjective responses from the participant, questionnaires also have serious methodological problems in this context [12]. Therefore questionnaires are best seen as supplements to behavioural and physiological data rather than the central means for assessment.

Physiological data, such as heart rate, heart rate variability and electrodermal activity provide excellent evidence of participant's physical reaction in real time (for example [9]). However, this is limited to a person's autonomic nervous system responses rather than higher level behavioural responses.

Here in addition we consider that the inclusion of actual bodily behaviour in our repertoire of possible factors could add significantly to our understanding people's responses, since they are clear and visible cues to which other people respond in turn. Several studies have used the behaviour of the participant in VE to evaluate presence $[11,14]$. One of these studies observed the participants actual responses to "danger", i.e. whether they ducked when virtual objects flew towards them [11]. More importantly, the interaction between human participants and virtual characters is crucial in many VE applications. In particular in therapeutic and training applications, where the involvement and behavioural response are the key factors, how the participant behaviour in the interaction is even more important than their reported "feeling" towards the virtual character. Few studies have examined the participants' interactive behaviour in VEs. Bailenson et al. observed the behaviour of participants towards a virtual character and found that they have showed greater hesitation in approaching the character which was with more human like movement [2]. Vinayagamoorthy et al. suggested that participants tended to adopt a socially-acceptable spatial be- 
haviour with virtual characters in immersive system [15]. Krämer assessed the verbal responses towards different interface and found that, when confronted with a virtual agent, the participants adopt more natural speech [6].

The paper examines the interpersonal behaviour of the participant while interacting with a virtual character in a virtual social encounter. Many important interpersonal communication factors were considered, such as: anxiety, domination, flirtation, affiliation, and avoidance.

\section{Methodology}

The work we present here is part of a larger experimental study carried out in an immersive (Cave) system, where shy and confident males interacted with a forward virtual woman, in a virtual bar [7]. In this paper we concentrate on male participants' bodily responses towards a virtual female in a virtual social encounter. Twenty-four participants were invited and attended the experiment. They were recorded with a camera from behind during the experiment and the recording were annotated afterwards by a body movement expert from UCLIC (UCL Interaction Centre) who otherwise had no involvement in the experiment and had no knowledge of the purpose of our research.

\subsection{Body Movement Annotations}

Compared to using motion capture for body movement annotation, manually annotating body movement through videos data has some own advantages. First, the results generated manually are more semanticly meaningful; secondly, the observation procedure is unobstrusive so that more spontaneous behaviours could be captured [4]. However, in our work, due to restrictions in the immersive projection system and the experimental setup, only video data taken from behind the participants was available for the annotation. As shown in Fig. 1, the video data was obtained with limited lighting condition and restricted view point. Therefore instead of using a standard annotation scheme we have formed our own body movement annotations which serves our purpose. Here we consider conversational behaviours which are related with anxiety, domination, flirtation, affiliation, and avoidance. We decomposed the body movement annotations into 3 categories: hand movement, head movement, and posture movement.

We included nodding, head cocking, head shaking, looking around, and looking down in the head movement analysis. Increased nodding and head cocking (see Fig.1(b)) shows affiliation and higher involvement, whilst looking around (Fig.1(d)) and looking down(Fig.1(e)) indicates lower involvement [1].

For hand movement we included: hands on hips, head-touching, hands in pockets, hands behind back, hands in front, hands making conversational gesture, and arm crossing. We are particularly interested in hands on hips (see Fig.1(f), 1(g), 1(h), and 1(i)), which is a dominant behaviour used by males while courting [1]. Also we considered head-touching, which is related to selfconsciousness within uncomfortable social situations [5]. Certain types of head- 
IV

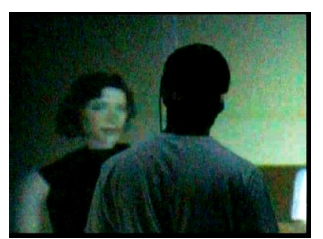

(a)

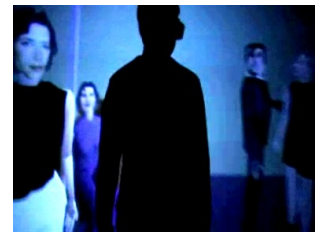

$(\mathrm{d})$

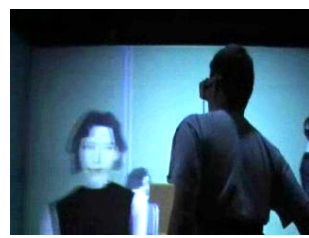

(g)

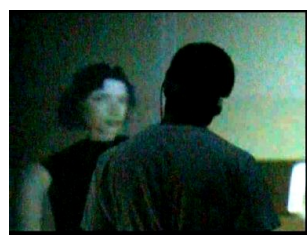

(b)

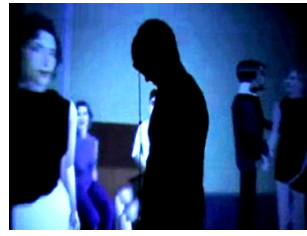

(e)

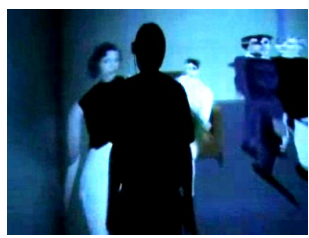

(h)

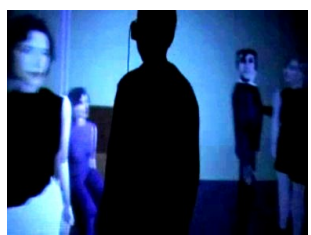

(c)

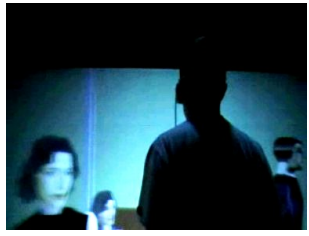

(f)

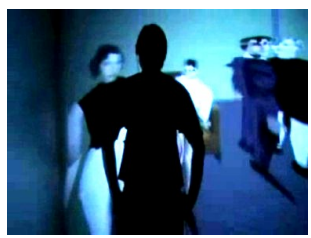

(i)

Fig. 1. (a) and (b) are the same participant where in (a) his head is straight and in (b) there is head-cocking; (c), (d) and (e) shows a participant with normal head position, looking around, and looking down. (f) and (h) show two participants' original positions, (g) and (i) show the same two participants with their hands on hips.

touching can also be interpreted as preening which is associated with courting; however, such behaviour is more common in women than men [1].

For posture movement, we look at posture shifting, shrugging, wiggling/swaying, and shifting weights. Similarly to head-touching, shifting weight reflects selfconsciousness related to uncomfortable social situation [5].

\subsection{Independent and Explanatory Variables}

There are four independent and explanatory variables considered. The first two factors are shy/confident and observed/not observed [7]. Additionally, because of the particular interaction generated by this experiment, another explanatory variable that might influence behaviour is the participant's reported relationship status. Therefore we consider the factor single/involved as one of the explanatory variables. During the experiment the virtual female asked the participant if he is involved or not with someone. The answers to this question were taken to determine their relationship status. Among the 24 participants, 12 of them reported themselves to be single, 12 of them were involved (we did not verify this with them afterwards). The last explanatory variable considered in this study is 
the changing level of intimacy as the conversation progressed. As shown in Table 1 , the whole interaction is segmented into 3 different periods where the level of intimacy changes: Approach, Mundane, and Intimate. With this segmentation we are able to monitor participants' behavioural changes as the conversation progresses.

Table 1. Sequence of Events and Virtual female Questions in the Virtual Encounter. Event here refers to a triggered utterance. It is segmented into 3 different period as the intimacy level increases.

\begin{tabular}{|l|l|}
\hline Approach: the virtual female initiates the conversation. \\
\hline 1 & Experiment starts and the Virtual female stares at the participant \\
\hline 2 & Virtual female stars approaching the participant \\
\hline 3 & "Hi, It looks like we are the only people alone here,right"? \\
\hline 4 & "My name is Christina." \\
\hline 5 & "So, what are you doing for a living?" \\
\hline 6 & "I'm an air hostess; I just arrived in London yesterday. Where do you live?" \\
\hline 7 & "I don't know London very well, but actually, I am thinking about moving \\
here, what do you think?"
\end{tabular}

\subsection{Assessing Body Movements}

On the assessment form a matrix is given defined by the 3 main periods as rows, and the columns defined by different body annotations. Each element of this matrix is the number of occurrences of a particular behaviour annotation at a certain period. The assessment form was given to the body movement expert who filled in it for each participant by screening the video data from the experiment; 10 hours were needed to conclude this task and she was paid for this work. 


\section{Results}

Consider any particular action such as 'head touching'. We were interested in whether there were any systematic variations of this response with the independent and explanatory variables of the experiment. The null hypothesis is that 'head touching' occurs at random through time. Under this null hypothesis the distribution the number of head-touches should for each individual follow a Poission distribution. Therefore we use the Poisson log-linear model as the appropriate model for analysis of variance of the response variable on the independent and explanatory variables.

In Table 2 we show the results of a series of such log-linear regressions. In each case the Poisson model fits well within the bounds of the traditional 5\% significance level, and we show the significant explanatory variables, whether their association with the response is positive or negative, and the corresponding significance level. These results show that:

- For head movement, participants who were observed by other virtual characters tended to look around more than participants who were not observed, and in the Mundane period participants tended to look around less than in the Approach period; shy participants tended to look down less than confident participants; in the Mundane period, participants tended to nod more than in the Approach period, and observed participants tended to nod less than participants who were not observed. In both Mundane and Intimate periods, participants tended to do more nodding and cocking than in the Approach period.

- For hand movement, participants who were observed tended to head-touch less, and single participants tended to head-touch more.

- For posture, observed participants tended to shift weights less than participants who were not observed.

Table 2. Variations of Response with Independent and Explanatory Variables.

\begin{tabular}{|c|c|c|c|c|}
\hline Categorise & Response Variable & \begin{tabular}{|l|} 
Explanatory \\
Variables \\
\end{tabular} & Association & $\begin{array}{l}\text { Significance } \\
\text { Level } \\
\end{array}$ \\
\hline \multirow{7}{*}{$\begin{array}{l}\text { Head } \\
\text { Movement }\end{array}$} & \multirow{2}{*}{$\begin{array}{l}\text { Looking } \\
\text { around }\end{array}$} & Observed & + & 0.00 \\
\hline & & Mundane & - & 0.05 \\
\hline & Looking down & Shy & - & 0.01 \\
\hline & \multirow[t]{2}{*}{ Nodding } & Mundane & + & 0.03 \\
\hline & & Observed & - & 0.06 \\
\hline & \multirow{2}{*}{$\begin{array}{l}\text { Nodding } \\
+ \text { Cocking }\end{array}$} & Mundane & + & 0.00 \\
\hline & & Intimate & + & 0.05 \\
\hline \multirow{2}{*}{\begin{tabular}{|l|} 
Hand \\
Movement
\end{tabular}} & \multirow[t]{2}{*}{ Head-touching } & Observed & - & 0.00 \\
\hline & & Single & + & 0.01 \\
\hline Posture & Shifting weights & Observed & - & 0.00 \\
\hline
\end{tabular}




\section{Discussion}

The results suggested that confident participants tended to look down more during the conversation. This might indicate that confident participants paid less attention to the virtual female, and were less involved with the interaction.

The results also show that participants who were observed by other virtual characters looked around more, and nodded, head-touched, and shifted weights less. The fact that they looked around more when being observed fits our expectation because it coincides with people's normal social behaviour of looking around when being observed by others. Less nodding, head-touching, and shifting weights furthermore suggest that participants who were observed may have been distracted and therefore paid less attention to the virtual female. However, in our previous analysis with only the questionnaire data and physiologically data, no difference was found between participants who were observed and those who were not.

Moreover, the result indicates that participants who are single head-touched more, which could be explained as greater attention being paid to the virtual female with more involvement in the conversation. This is also a factor that failed to stands out with other measurements in our previous work.

Finally, compared to the Approach period, there is more nodding and head cocking in Mundane and Intimate periods. Also in Mundane period the participants looked around less. All these results suggest that the participants became more involved in the interaction as the conversation progressed, which coincides with our physiological results.

\section{Conclusion and Future work}

The results support the notion that participants tended to act towards the virtual character with appropriate interpersonal behaviour. Body movement is a gross overall indicator of a person's state, and is relatively easily observable. It therefore could offer us additional methods to measure the responses of people in VEs, especially when the participant interacts with a virtual character. In our previous report [7] we evaluated the reactions of participants and the results showed that the participants tended to respond to the situation at the subjective and physiological level as if it were real. In the previous study we have also evaluated their verbal responses to assess their behaviour, yet one can argue that verbal responses can be playfully delivered by the participants without being serious. This new evaluation of bodily responses, however, underlines the findings of our previous study, since it is unlikely that people deliberately and consciously choose their bodily responses - they are an automatic action. Another contribution of this work is that a bodily annotation system which focuses on conversational behaviours was proposed for annotating body movement through video data with poor view and lighting conditions. In further research, the analysis of facial expressions, voice and other automatic human responses is being considered. These results further emphasise that virtual reality technology can be used in the treatment of social phobia. 


\section{Acknowledgments}

This work is funded through the EPSRC Empathic Avatar project EP/D505542/1. We would like to thank Andrea Kleinsmith from UCLIC for her professional help in the annotation of body movement.

\section{References}

1. Michael Argyle. Bodily Communication. Methuen \& Co Ltd, second edition, 1988.

2. J. N. Bailenson, K. R. Swinth, C. L. Hoyt, S. Persky, A. Dimov, and J. Blascovich. The independent and interactive effects of embodied agent appearance and behavior on self-report, cognitive, and behavioral markers of copresence in immersive virtual environments. In PRESENCE, 2005.

3. D. Freeman, K. Pugh, A. Antley, M. Slater, P. Bebbington, and M. Gittins. A virtual reality study of paranoid thinking in the general population. pages $627-$ $633,2008$.

4. M. Kipp, M. Neff, and I. Albrecht. An annotation scheme for conversational gestures: how to economically capture timing and form. Language Resources and Evaluation, December.

5. M.L. Knapp. Nonverbal communication in human interaction. Holt, Rinehart and Winston New York, 1978.

6. N. Krämer. Social communicative effects of a virtual program guide. In IVA, 2005.

7. X. Pan and M. Slater. A preliminary study of shy males interacting with a virtual female. In Presence: The 10th Annual International Workshop on Presence, 2007.

8. M V Sanchez-Vives and Mel Slater. From presence to consciousness through virtual reality. Nature Reviews Neuroscience, 6(4):332-339, 2005.

9. M. Slater, A. Antley, A. Davison, D. Swapp, C. Guger, and C. Barker. A virtual reprise of the stanley milgram obedience experiments. PLoS ONE, 1(1):e39, 2006.

10. M. Slater, D. P. Pertaub, C. Barker, and D. M Clark. An experimental study on fear of public speaking using a virtual environment. 9(5):627-633, 2006.

11. M. Slater and M Usoh. Representations systems, perceptual position, and presence in immersive virtual environments. In Presence: Teleoperators and Virtual Environments, 1993.

12. Mel Slater. How colourful was your day?: Why questionnaires cannot assess presence in virtual environments. Presence: Teleoperators and Virtual Environments, 13(4):484-493, 2004.

13. Mel Slater, Amela Sadagic, Martin Usoh, and Ralph Schroeder. Small group behaviour in a virtual and real environment: A comparative study. Presence: Teleoperators and Virtual Environments, 9(1):37-51, February 2000.

14. S. Uno and M. Slater. The sensitivity of presence to collision response. In Virtual Reality Annual International Symposium, 1997.

15. Vinayagamoorthy V., Brogni A., Steed A., and Slater M. The role of posture in the communication of affect in immersive virtual environments. In The 2nd ACM SIGGRAPH International Conference on Virtual Reality Continuum and Its Applications, 2006.

16. B Witmer and M Singer. Measuring presence in virtual environments: a presence questionnaire. Presence, 7(3):225-240, 1998. 